\title{
THE TEXTURE OF THIN NiSi FILMS AND ITS EFFECT ON AGGLOMERATION
}

\author{
K. De Keyser, C. Detavernier, R. L. Van Meirhaeghe \\ Department of Solid State Physics, Ghent University \\ Krijslaan $281 \mathrm{~S} 1$ \\ Gent, 9000 \\ Belgium
}

J. Jordan-Sweet, C. Lavoie

IBM T.J. Watson Research Center

Yorktown Heights

New York, 10598

United States of America

\section{ABSTRACT}

Nickel silicide films are used as contacting materials in the micro electronics industry. It was recently [1] discovered that these films exhibit a peculiar type of texture, which was called 'axiotaxy', whereby certain lattice planes in the NiSi grains are preferentially aligned to (110)type lattice planes in the single crystal $\mathrm{Si}$ substrate. In this contribution, we present a quantitative study of this phenomenon, using both XRD pole figure measurements and EBSD. Furthermore, we report a correlation between the texture of these NiSi films and their morphological stability during annealing at high temperature.

In spite of the small grain size in these films, EBSD could be used to determine the volume fractions of the various texture components. This provided quantitative support for the claim that axiotaxy is the main texture component in these films, as about $40 \%$ of the grains belong to one of the axiotaxial texture components, and the remaining fraction exhibits a random orientation. A discussion of the techniques used during the measurement and analysis of the EBSD data is presented, as this must be given special consideration in view of the peculiar type of texture encountered in these films.

Secondly, both XRD and EBSD were performed after annealing the NiSi films at various temperatures and durations. It is known that thin $\mathrm{NiSi}$ films have a strong tendency to agglomerate [2]. Our data indicates a correlation between the texture evolution and the agglomeration of the NiSi layer. Grains with axiotaxial orientation were observed to grow and thicken during the annealing process, by consuming neighboring randomly oriented grains. This suggests that the texture of the NiSi layer is a determining factor for the morphological stability of the film. The fact that grains with axiotaxial orientation grow during heat treatment can be related to the one dimensional periodicity at the interface, which lowers the interface energy and thus provides a driving force for the preferred growth of these grains. The agglomeration of NiSi films results in a significant increase of the sheet resistance. Therefore, these results illustrate the importance of texture control for the application of these films as contacts in micro-electronic devices.

\section{INTRODUCTION}

Silicides play a vital role in the construction of micro-electronic devices [3]. They are typically used as contacting materials on gate, source and drain regions in the CMOS process and 
also show promise in new applications such as fully silicided (FUSI) gates [4], and nano-wires [5]. Historically, three silicides have been used in micro-electronics as contacting materials, due to their low resistivity: initially, $\mathrm{TiSi}_{2}$ was used, but due to the difficulty of growing the low resistivity $\mathrm{C} 54$ phase of $\mathrm{TiSi}_{2}$ in narrow lines, the transition to $\mathrm{CoSi}_{2}$ was made. Further miniaturization lead to the adoption of $\mathrm{NiSi}$ as the currently used contacting material in deep submicron technology [3].

The viability of the use of a specific silicide depends on multiple parameters: it has to be possible to reliably grow the silicide in narrow lines, using a limited thermal budget (to insure compatibility with the other process steps), silicon consumption cannot be too high, and the silicide needs to stay stable and not degrade during all of the following process steps. The first and second problem could only be avoided by adopting NiSi as contacting material, however the third problem has been shown to be still significant. In contrast to $\mathrm{CoSi}_{2}$ and $\mathrm{TiSi}_{2}$, the $\mathrm{NiSi}$ phase is not the end-phase of the metal-silicon binary system ( $\mathrm{NiSi}_{2}$ is), which, when further heating a NiSi contact in contact with the silicon substrate, could lead to a conversion of the contact to the higher resistivity $\mathrm{NiSi}_{2}$ phase, resulting in poor device performance. For thin NiSi layers, such as those typically used in the current deep submicron processes, it has been shown that this is only a secondary problem, as the thin NiSi film tends to break up and agglomerate first, long before it is converted into the $\mathrm{NiSi}_{2}$ phase [2]. As agglomeration leads to a huge increase in the resistivity of the contact, this has to be avoided. It has been shown that the addition of alloying elements to the NiSi layer can delay the agglomeration, however, the physical reason for this delay is not completely understood.

\section{TEXTURE OF SILICIDES}

Until recently, the texture of silicides was generally considered to be either random, or epitaxial. Indeed, a lot of attention has been given to the effort of growing epitaxial silicides, such as $\mathrm{CoSi}_{2}$ and $\mathrm{NiSi}_{2}$, both cubic, which, due to the close matching of their lattice parameter to the cubic Si crystal lattice size, can be grown quite well epitaxially [6]. For the non-cubic phases, such as $\mathrm{TiSi}_{2}$ and $\mathrm{NiSi}$, an epitaxial match between film and substrate is less obvious. Based on X-ray diffraction measurements in Bragg-Brentano geometry, it was concluded that these materials exhibit a random texture [7]. Indeed, lots of peaks, belonging to different planes, show up, indicating that not one single specific plane is parallel to the substrate. From this, one might jump to the conclusion that the film is thus randomly textured. However, looking at the intensities of the peaks indicates a discrepancy with the structure factors of the silicide. Measuring pole figures provided more information about texture. For $\mathrm{CoSi}_{2}$, this was done by Bulle-Lieuwma et al. [8], resulting in a variety of epitaxial orientations in which $\mathrm{CoSi}_{2}$ can grow on $\mathrm{Si}$ 100. For NiSi, this resulted in the discovery of a new type of texture in thin films, which was given the name "axiotaxy" [1].

Texture of NiSi: axiotaxy

An XRD pole figure for a 60nm NiSi film, recorded at the X20A beam line at the National Synchrotron Light Source, can be seen in figure 1a. Several arcs are visible, which can be explained by assuming that the grains making up the film have certain planes preferentially parallel to planes in the substrate. For NiSi, 4 sets of planes were identified as having a preferential orientation (table I). This alignment is called axiotaxy and results in an interface which is periodic along one direction, and axiotaxy can thus be considered as in-between random (no periodicity) and epitaxy (periodicity along multiple directions) In addition to the four 
axiotaxies, several spots are visible, indicating the existence of an additional epitaxial component in the texture.
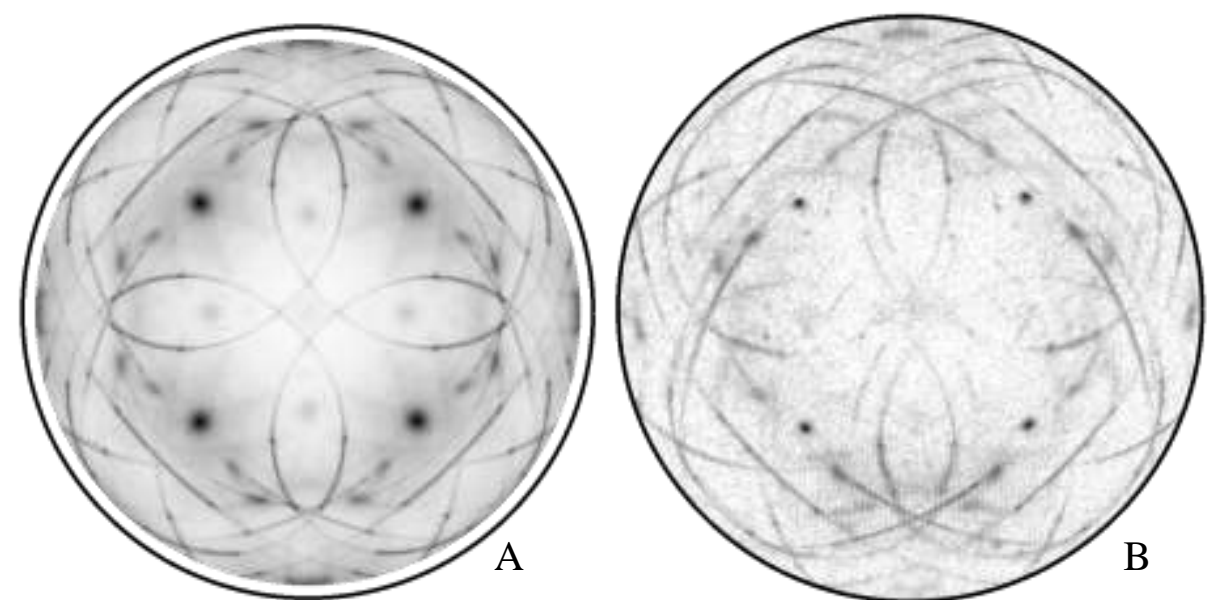

Figure 1: (202)+(211) pole figures of 60nm NiSi films on Si 100. XRD (left, A) EBSD using GUSTAV (right, B)

Table I: texture components in NiSi films on Si 100

\begin{tabular}{|c|c|}
\hline Texture name & Matching planes \\
\hline$(202)$ axiotaxy & $\mathrm{NiSi}(202) / / \mathrm{Si}(220)$ \\
\hline$(211)$ axiotaxy & $\mathrm{NiSi}(211) / / \mathrm{Si}(220)$ \\
\hline$(103)$ axiotaxy & $\mathrm{NiSi}(103) \sim / / \mathrm{Si}(220)$ \\
\hline$(112)$ axiotaxy & $\mathrm{NiSi}(112) \sim / / \mathrm{Si}(220)$ \\
\hline epitaxy & $\mathrm{NiSi}(029) / / \mathrm{Si}(100)$ and $\mathrm{NiSi}(100) / / \mathrm{Si}(010)$ \\
\hline
\end{tabular}

The analysis of the texture of NiSi on Si (100), based on XRD pole figures, allowed the qualitative identification of the different texture components, however, getting quantitative information from these pole figures proves much more difficult. Pole figures can contain information from multiple planes if these planes have similar d-spacings, including planes from the substrate, and the very sharply defined features (i.e. axiotaxy) and low symmetry materials, make the use of standard ODF calculation tools impossible.

\section{EBSD on NiSi}

To get an idea of the relative importance of the different axiotaxy components, electron backscatter diffraction (EBSD) measurements were performed using an FEI Quanta 200F field emission SEM. Two types of measurements were carried out. A first type of scan was a typical EBSD map scan, where several points are collected in each of the grains, by using a step size which is considerably smaller than the grain size in the material. From these measurements, spatial information can be retrieved, such as grain boundaries or the location of grains with a specific orientation. For this measurement, the samples were first cleaned in a plasma oven in an $\mathrm{Ar}-\mathrm{O}_{2}$ mixture, to remove carbon contamination. Maps were then measured using step sizes of $20 \mathrm{~nm}$ and bigger. Only conclusions with limited reliability could be drawn from this data. Indeed, if one compares the statistics of the typical measurement techniques for texture in thin films, this problem becomes clear. Using XRD and a spot size of $1 \mathrm{~mm}^{2}$, recording a pole figure on a sample with an average grain size of about $100 \mathrm{~nm}$, results in information from $10^{8}$ grains. A typical EBSD map, using a step size of 20nm, and a measurement grid of 1000x1000 data points, 
gives information about only $10^{4}$ grains, indicating that the statistics of this measurement are much worse than in the case of the XRD pole figures. This is confirmed when calculating pole figures from EBSD map measurements and comparing them to the XRD ones: the axiotaxy lines are hardly visible. A third technique, using TEM, results in even worse statistics, only a few grains can be investigated. For our goal of getting an overview of the texture, this was of limited use.

To improve the statistics in the case of the EBSD measurement, a second type of scan was devised, which we called an "EBSD pole figure scan". Here, a large grid is measured, using a large step size. As the step size is as big as, or bigger than the grain size in the film, each point is sampled in a different grain, resulting in orientation information for $10^{5-1} 10^{6}$ grains. This strategy was carried out during multiple measurements on NiSi films of different thickness.

Ni was sputter deposited on RCA cleaned and HF dipped Si 100 wafers, with a thickness of 45 and $30 \mathrm{~nm}$. They were annealed at $550^{\circ} \mathrm{C}$ for 30 seconds, and then cooled down to room temperature. This results in the formation of either 90 or $60 \mathrm{~nm}$ of NiSi. Both traditional EBSD map measurements, as well as "pole figure measurements" consisting of 420000 data points (EBSD patterns) with a step size of $250 \mathrm{~nm}$, were collected for each of the samples, using an HKL Channel 5 system. Pole figures were calculated from this data, using both the HKL software, as well as the GUSTAV software, an in-house developed software package for texture analysis of both XRD and EBSD data. Figure 2a shows an EBSD map of the 60nm sample. The grains belonging to each of the texture components of table I were identified. They are indicated in color on figure $2 \mathrm{~b}$. Figure $1 \mathrm{~b}$ shows the $(202)+(211)$ pole figure which was calculated from the EBSD data using the GUSTAV software. It has an excellent resemblance to the XRD one shown in figure 1a. It is worth noting that the excellent resemblance is only visible on the pole figures calculated using GUSTAV, as the fine curves, with relatively low intensity (total intensity of the axiotaxy is spread out along the line, while all the intensity of an epitaxial texture component contributes to a single point in the pole figure) easily gets lost in the coarse grid (pole figure is divided in cells with a size of multiple degrees in chi and phi) used by commercial EBSD software, which often has limited options to choose appropriate intensity scales too.

(a)

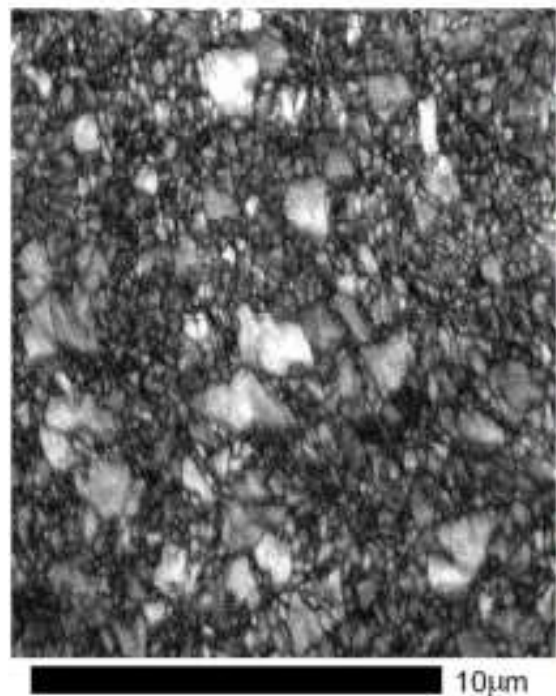

(b)

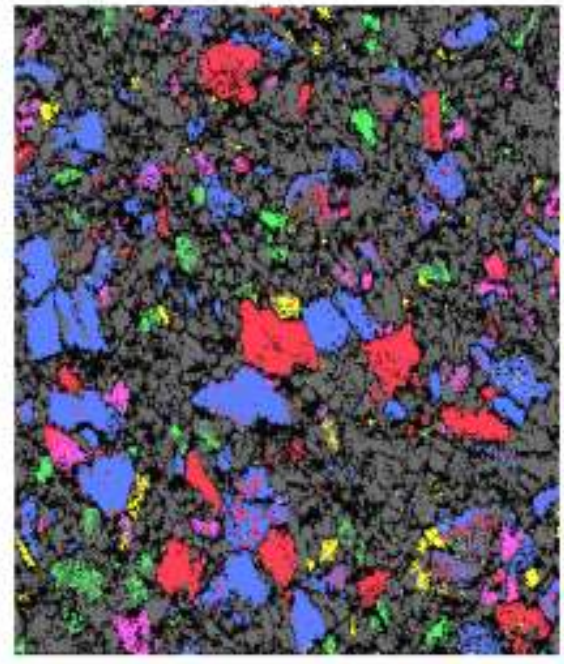

$\square$ no data
$\square$ epitaxy

$\square(112)$ axiotaxy

$\square$ (103) axiotaxy

(202) axiotaxy

(211) axiotaxy

random

Figure 2: EBSD map on 60nm NiSi film. (a) shows band contrast, (b) shows texture components 
From the EBSD measurements, volume fractions for all of the texture components could be calculated [9], the results for which can be found in table II. It is important to stress that the absolute numbers for the volume fractions based on EBSD, when carried out on materials with small grains and low symmetry, have a limited reliability, as the software used to analyze these EBSD patterns tends to introduce errors, especially on lower symmetry materials like NiSi (orthorhombic). Furthermore, there is a slight dependence of the volume fraction on the settings of the microscope, such as acceleration voltage and spot size. Still, the numbers confirm that axiotaxy is an important part (about 40\%) of the texture of the thin NiSi films, however it also shows that randomly oriented grains make up an important part of the film as well.

Table II: volume fractions (in \%) from EBSD measurements on 60 and 90nm NiSi films

\begin{tabular}{|c|c|c|c|}
\hline Texture Component & $60 \mathrm{~nm} \mathrm{NiSi}$ & $90 \mathrm{~nm} \mathrm{NiSi}(1)$ & 90nm NiSi (2) \\
\hline$(211)$ axiotaxy & 19 & 16 & 15 \\
\hline (202) axiotaxy & 17 & 17 & 15 \\
\hline (103) axiotaxy & 5 & 4 & 4 \\
\hline (112) axiotaxy & 3 & 2 & 2 \\
\hline Epitaxy & 3 & 2 & 2 \\
\hline
\end{tabular}

\section{AGGLOMERATION OF THIN NiSi FILMS}

The integration of NiSi in the standard CMOS process faces an important problem: thin films $(<50 \mathrm{~nm})$ of NiSi start to agglomerate at quite low temperatures $\left(650^{\circ} \mathrm{C}\right)$. This phenomenon has been studied [2], and it has been shown that the addition of alloying elements can postpone this agglomeration to much higher temperatures [10]. In particular, the addition of Pt to a NiSi layer seems to prevent this quite well [11]. A number of suggestions have been made regarding the mechanism by which Pt prevents this: a change in texture in $\mathrm{NiPt}_{\mathrm{x}} \mathrm{Si}$ films compared to NiSi was reported, hinting at an effect of Pt on the texture, and calculations based on a phase-field model suggest that $\mathrm{Pt}$ moves to the grain boundaries of the NiSi film and prevents these boundaries from moving [12], however, the assumption in the latter model of random texture in the NiSi film is in our case clearly not valid.

Ni films with a thickness of 10 and $30 \mathrm{~nm}$ were sputter deposited on HF cleaned Si 100 substrates, resulting in 20 and 60nm NiSi films after annealing. The films with 30nm Ni received a $2 \mathrm{~nm}$ Si cap. They were investigated using EBSD and XRD pole figures (X20A, NSLS). On the 20nm NiSi samples, EBSD proved impossible, due to the small grain size and thin films. XRD pole figures were successfully recorded on the $20 \mathrm{~nm}$ NiSi samples, ramp annealed to either 500, $650,700,800$ and $840^{\circ} \mathrm{C}$. Figure 3 shows the evolution of the texture of the film in an (112) pole figure. All pole figures are plotted on the same, logarithmic, scale. For left to right (= increasing temperature) one sees a clear evolution in the texture of the film. At $700^{\circ} \mathrm{C}$, the intensity in certain regions of the pole figures has significantly changed, and the axiotaxy lines increase in intensity. Further heating to $840^{\circ} \mathrm{C}$ results in a significant increase of intensity in the epitaxial spots, and a lower random background. Since an (hkl) type of axiotaxy reduces to a spot in an (hkl) pole figure, the number of XRD counts in this spot is proportional to the amount of material having the axiotaxial orientation, and can be used to get an estimate of the importance of a specific axiotaxy texture component. However, for the (202), (211) and (112) axiotaxy, the dspacing of those NiSi planes is close to the one of the Si (220) plane, making it impossible to isolate the XRD signal of NiSi from the Si (220) signal, since they both result in spots at the same location in the pole figure. For NiSi (103), this problem does not occur, and figure 4 shows 
the X-ray intensity, corrected for background, in the (103) fiber axis, as a function of annealing temperature. The intensity stays constant up until $650^{\circ} \mathrm{C}$, after which a sharp increase is visible. If one compares this to the resistance measurements in reference [2], this can be correlated to the sharp increase in sheet resistance of NiSi films between $650-700^{\circ} \mathrm{C}$. We can conclude that the increase of the resistance is mostly likely related to the change in texture of the material. Combining the information from figure 3 and 4, this suggests that the random oriented grains will disappear during agglomeration, in favor of epitaxial and axiotaxial grains.
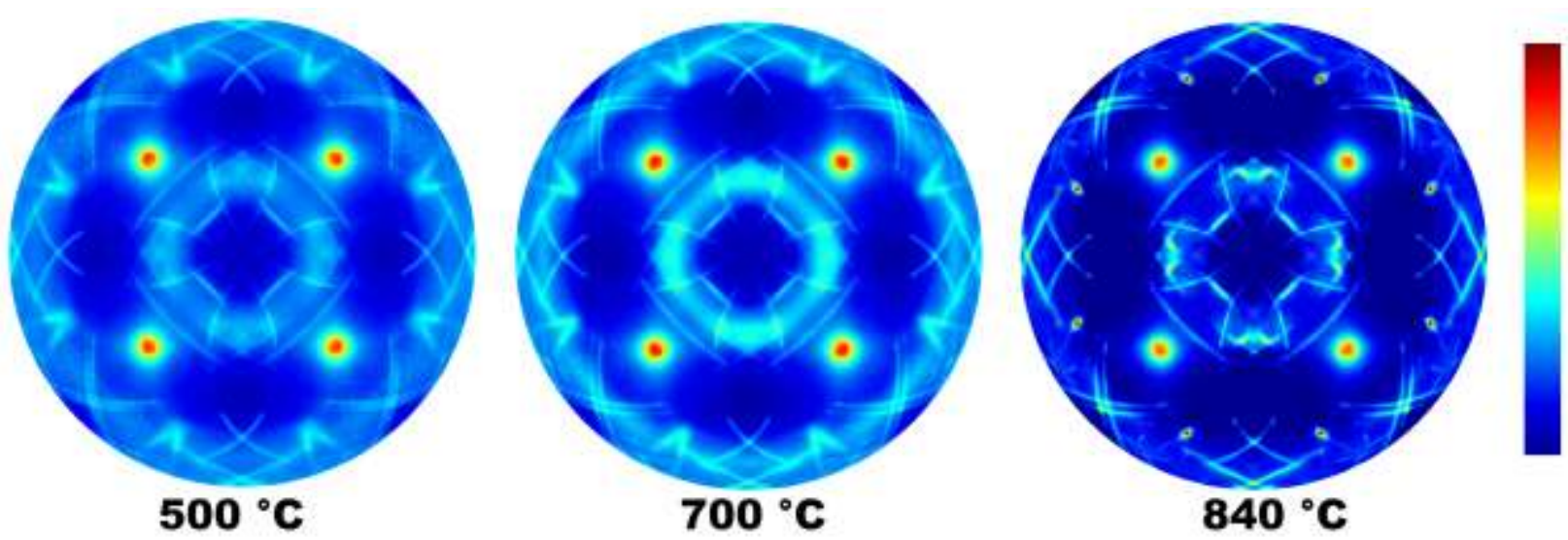

Figure 3: XRD (112) pole figures on 20nm NiSi films at different temperatues

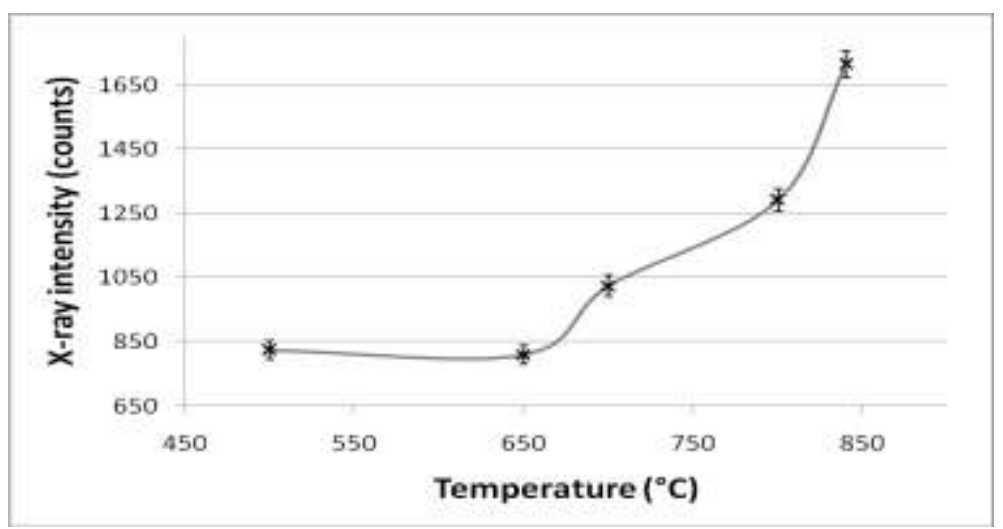

Figure 4: X-ray intensity for (103) axiotaxy as function of annealing temperature

As EBSD measurements on the 20nm NiSi samples were impossible, we also looked at the thicker $60 \mathrm{~nm}$ NiSi layers. Due to their increased thickness, agglomeration will be much more difficult, and the layer will first convert to $\mathrm{NiSi}_{2}$ when heating to higher temperatures $\left(700^{\circ} \mathrm{C}\right)$. To prevent the $\mathrm{NiSi}_{2}$ growth, the $60 \mathrm{~nm} \mathrm{NiSi}$ layers were annealed at a lower temperature, $550^{\circ} \mathrm{C}$, for different time periods. Agglomeration proved to be very slow, and even after a $72 \mathrm{~h}$ anneal, the films were only starting to agglomerate. On the samples, EBSD measurements were carried out using 200000 data points, always using identical settings, both in hardware and software (Channel 5, Advanced Indexing). The volume fractions for each of the axiotaxy texture components were calculated, both for the sample spike annealed to $550^{\circ} \mathrm{C}$ with no dwell time, as for a sample with a dwell time of $72 \mathrm{~h}$ at $550^{\circ} \mathrm{C}$. In table III, the volume fractions are indicated. 
Even though agglomeration has only started, we can already notice an increase in the volume fractions for the axiotaxial grains for $24 \%$ to $30 \%$, supporting the results on the $20 \mathrm{~nm} \mathrm{NiSi}$ samples. The difference in the absolute values for the volume fractions with table I are most likely caused by the addition of a $2 \mathrm{~nm} \mathrm{Si}$ cap, to prevent the Ni from oxidizing. XRD pole figures were recorded as well (figure 5). Plotted on the same scale, the increase of the axiotaxial and epitaxial components after a $72 \mathrm{~h}$ anneal is clearly visible.
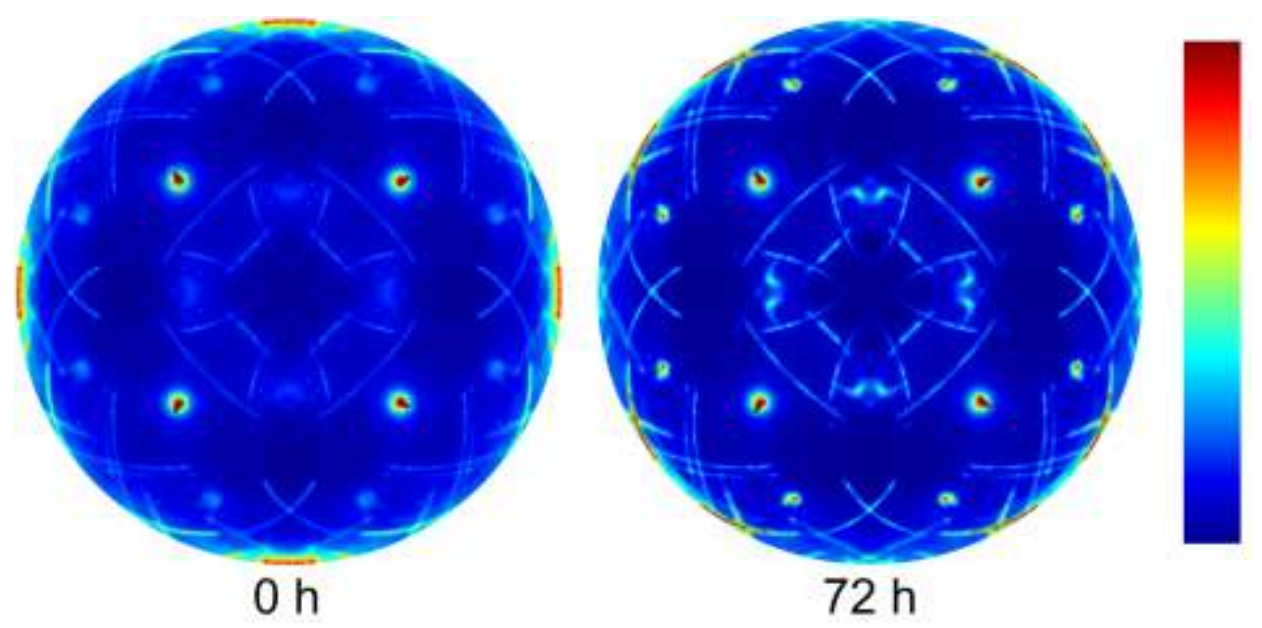

Figure 5: (112) pole figures on $60 \mathrm{~nm}$ NiSi films, spike annealed to $550^{\circ} \mathrm{C}$ with a dwell time of $0 \mathrm{~h}$ (left) or $72 \mathrm{~h}$ (right).

Table III: volume fractions (in \%) from EBSD measurements on $60 \mathrm{~nm}$ NiSi films, ramp annealed to $550^{\circ} \mathrm{C}$ with a dwell time of $0 \mathrm{~h}$ or $72 \mathrm{~h}$.

\begin{tabular}{|c|c|c|}
\hline Texture Component & Oh dwell time & 72h dwell time \\
\hline (211) axiotaxy & 9 & 11 \\
\hline (202) axiotaxy & 10 & 12 \\
\hline$(103)$ axiotaxy & 3.5 & 5.5 \\
\hline$(112)$ axiotaxy & 1.5 & 1.5 \\
\hline total axiotaxy & 24 & 30 \\
\hline
\end{tabular}

Mechanism for agglomeration

Since epitaxial grains have an excellent interface match with the substrate, and axiotaxial grains, while slightly worse than epitaxy, still have a decent interface match due to the 1dimensional periodicity, there is a driving force for axiotaxial and epitaxial grains to consume randomly oriented neighbor grains, due to the lowering of the interface energy which can be accomplished in that way. As noted in [1], the axiotaxial orientation relation has the property of being largely independent of the shape of the interface. Going from a flat interface to a spherical one results in the lowest amount of interface energy per unit of volume of the grain. By consuming random grains and curving the interface, axiotaxial grains both reduce the interface energy per unit of surface (going from zero- to one-dimensional periodicity at the interface), as well as the total surface at the interface (due to the curving of this interface). This was confirmed by TEM measurements in [2], were axiotaxy grains are shown to have a curved interface in agglomerated films. For epitaxial grains, the typical plane-on-plane matching depends heavily on the shape of the interface with the substrate. Curving results in a loss of the low energy epitaxial interface, thus the epitaxial grains can only increase their size by growing laterally, and keeping 
the interface plane flat. As agglomeration is in fact nothing more than a thickening and curving of grains, the occurrence of axiotaxial grains will thus promote the agglomeration.

From the pole figures in $[10,11]$, it is clear that adding alloying elements changes the texture of the material, by suppressing the axiotaxial grains and promoting the epitaxial ones. In light of our results, it becomes evident why this delays agglomeration.

\section{CONCLUSION}

We have shown the possibility of investigating the texture of silicides using EBSD, resulting in information on volume fractions for the different texture components. Using XRD pole figures and EBSD, we have studied the evolution of the texture in thin NiSi films during agglomeration and have found evidence for a correlation between axiotaxy and tendency to agglomerate. From these results, we were able to explain the effect of alloying elements on the agglomeration behavior.

\section{REFERENCES}

${ }^{1}$ C. Detavernier, A. S. Ozcan, J. Jordan-Sweet, E.A. Stach, J. Tersoff, F.M. Ross, C. Lavoie, "An off-normal fibre-like texture in thin films on single-crystal substrates." Nature 426, 641-645 (2003)

2 D. Deduytsche, C. Detavernier, R.L. Van Meirhaeghe, C. Lavoie, "High-temperature degradation of NiSi films: Agglomeration versus NiSi2 nucleation." J. Appl. Phys. 98(3), 033526 (2005)

3 S.L. Zhang, M. Östling, "Metal Silicides in CMOS Technology: Past, Present , and Future Trends", Crit. Rev. Solid State Mater. Sci., 28(1), 1-129 (2003)

${ }^{4}$ J.A. Kittl, M. A. Pawlak, et al, "Transient and end silicide phase formation in thin film Ni/polycrystalline-Si reactions for fully silicided gate applications." Appl. Phys. Lett. 91(17), 172108 (2007)

${ }^{5}$ C.A. Decker, R. Solanki, J.L. Freeouf, J. Carruthers, D.R. Evans, "Directed growth of nickel silicide nanowires." Appl. Phys. Lett. 84(8), 1389-1391 (2004)

${ }^{6}$ L.J. Chen, J. W. Mayer, K.N. Tu, "Formation and structure of epitaxial NiSi2 and CoSi2", Thin Solid Films, 93, 135-141 (1982)

${ }^{7}$ F. Dheurle, C. S. Petersson, J.E.E. Baglin, S.J. Laplaca, C.Y. Wong, "Formation of thin films of NiSi - Metastable structure, diffusion mechanisms in intermetallic compounds", J. Appl. Phys., 55(12), 4208-4218 (1984)

${ }^{8}$ C. W. T. Bullelieuwma, A. H. Vanommen, J. Hornstra, C. Aussems, "Observation and analysis of epitaxial growth of $\mathrm{CoSi}_{2}$ on (100) Si”, J. Appl. Phys. , 71 (5), 2211-2224 (1992)

${ }^{9}$ K. De Keyser, C. Detavernier, R.L. Van Meirhaeghe, "Characterization of the texture of silicide films using electron backscattered diffraction", Appl. Phys. Lett., 90(12), 121920 (2007)

${ }^{10}$ D. Deduytsche, C. Detavernier, R.L. Van Meirhaeghe, J.L. Jordan-Sweet, C. Lavoie, "Formation and morphological stability of $\mathrm{NiSi}$ in the presence of $\mathrm{W}$, Ti, and $\mathrm{Ta}$ alloying elements." J. Appl. Phys. 101(4) 044508 (2007)

${ }^{11}$ C. Detavernier, C. Lavoie, "Influence of Pt addition on the texture of NiSi on $\mathrm{Si}(001) . "$, Appl. Phys. Lett., 84(18), 3549-3551 (2004)

${ }^{12}$ M. Bouville, S. Y. Hu, L.Q. Chen, D.Z. Chi, D.J. Srolovitz, "Phase-field model for grain boundary grooving in multi-component thin films.", Modell. Simul. Mater. Sci. Eng., 14(3), 433-443 (2006) 